\title{
Editorial
}

\section{Preserver Problems on Function Spaces, Operator Algebras, and Related Topics}

\author{
Antonio M. Peralta, ${ }^{1}$ Chi-Keung Ng, ${ }^{2}$ Ngai-Ching Wong, ${ }^{3}$ and Jen-Chih Yao ${ }^{4}$ \\ ${ }^{1}$ Departamento de Análisis Matemático, Facultad de Ciencias, Universidad de Granada, 18071 Granada, Spain \\ ${ }^{2}$ Chern Institute of Mathematics, Nankai University, Tianjin 300071, China \\ ${ }^{3}$ Department of Applied Mathematics, National Sun Yat-sen University, Kaohsiung 804, Taiwan \\ ${ }^{4}$ Center for Fundamental Science, Kaohsiung Medical University, Kaohsiung 807, Taiwan
}

Correspondence should be addressed to Antonio M. Peralta; aperalta@ugr.es

Received 21 January 2014; Accepted 21 January 2014; Published 27 February 2014

Copyright (C) 2014 Antonio M. Peralta et al. This is an open access article distributed under the Creative Commons Attribution License, which permits unrestricted use, distribution, and reproduction in any medium, provided the original work is properly cited.

This special volume of the journal Abstract and Applied Analysis was originally conceived to provide authors and readers a publication to present the most recent advances in the study of those (linear) mappings preserving ranks, spectra, spectral radii, numerical ranges, numerical radii, orthogonality, or disjointness between different, though related, structures studied in Matrix theory, Functional Analysis, and Operator theory, including Function spaces, Operator algebras, and $C^{*}$-algebras.

This volume includes 23 original research papers by 66 authors. The selection of the papers included in this volume, which has been based on a strict international peer review procedure, contains a representative list of manuscripts with newfangled results which covers the different topics considered in our original proposal.

In the setting of preservers between $C^{*}$-algebras and Matrix spaces, the volume includes a characterization of $\xi$-Lie multiplicative isomorphisms between von Neumann algebras by Y. Song et al.; a study on linear maps on upper triangular matrices spaces preserving idempotent tensor products by L. Yang et al. M. Burgos et al. study approximate versions of Hua's theorem and other related results for Banach algebras and $C^{*}$-algebras, including a study of linear maps approximately preserving the conorm between unital $C^{*}$-algebras. Pioneering achievements on orthogonally additive and orthogonality preserving holomorphic mappings between $C^{*}$-algebras together with a newfangled investigation on orthogonality preserving pairs of operators between $C^{*}$-algebras are presented by J. J. Garcés et al. A study on linear maps between positive operators of Schatten $p$-classes $(1<p<+\infty)$ which preserve the $p$-norms of convex combinations is developed by D. L.-W. Kuo et al. New methods and results to solve systems of operator equations on Hilbert $C^{*}$-modules are given by $\mathrm{X}$. Fang et al.

Y. Song et al. characterize all maps between von Neumann algebras that satisfy the equality $\Phi(a b-\xi b a)=\Phi(a) \Phi(b)-$ $\xi \Phi(b) \Phi(a)$. M. A. Alghamdi et al. obtain basic properties of the solutions of the Q-lasso as a function of a tuning parameter $\gamma$. They also discuss iterative methods for solving the Q-lasso which include the proximal gradient algorithm and the projection-gradient algorithm. L. Yang et al. characterize, for a general field $F$ and $m, n \geq 3$, maps of the form $\left\{f_{i j}\right\}$ from $M_{m n}(F)$ to itself that preserves similarity and those that preserves inverses.

Concerning the applications of the fixed point theorems and various techniques developed in preserver problems, $\mathrm{H}$. Liu et al. establish some existence theorems for semilinear nonlocal functional differential equations. D.-G. Yang and J.L. Liu study new subclasses of multivalent analytic functions associated with a linear operator. Moreover, T. Wang and F. Y. Lu obtain some new results on Lie triple derivations on standard subalgebras of lattice algebras. 
The paper by L. C. Ceng and C. F. Wen proposes and analyzes hybrid implicit and explicit extragradient methods for finding a zero of an accretive operator and solving a general system of variational inequalities and a fixed point problem of an infinite family of nonexpansive self-mappings in a uniformly convex Banach space $X$ which has a uniformly Gateaux differentiable norm. Several strong convergence theorems for hybrid implicit and explicit extragradient algorithms under suitable assumptions are established. An implicit algorithm for the split fixed point and convex feasibility problems is proposed in the paper by F. Guo et al. A strong convergence theorem is obtained. The paper by Y. Ling et al. is concerned with the semilocal as well as the local convergence problems of Newton-Steffensen's method to solve nonlinear operator equations in Banach spaces. Under very mild assumptions, they establish the convergence criterion and convergence ball for NewtonSteffensen's method.

Mann-type extragradient methods for a general system of variational inequalities with solutions of a multivalued variational inclusion and common fixed points of a countable family of nonexpansive mappings in real smooth Banach spaces are introduced in the paper by L. C. Ceng et al. Weak and strong convergence results are derived. The paper by $\mathrm{N}$. Hussain et al. introduces modified weak $\alpha-\psi$-contractive mappings, where fixed point results for such mappings defined on partial metric spaces using the notion of triangular $\alpha$-admissibility are obtained. The well-posedness for generalized set equilibrium problems is studied in the paper by Y. C. Lin, where two types of well-posed concepts for these problems in topological vector spaces setting are proposed and the relationship between these well-posednesses is obtained.

The paper by M. Alshahrani et al. introduces nonsmooth vector quasi-variational-like inequalities by means of a bifunction, where some existence results for solutions of these inequalities are established by using Fan-KKM theorem and a maximal element theorem. The paper by D. H. Fan and J. F. Bao considers a generalized equilibrium problem involving DC functions. By using the properties of the epigraph of the conjugate functions, they provide some sufficient and/or necessary conditions for the weak and strong duality results and optimality conditions for generalized equilibrium problems. The paper by Z. Chen and Zhe Chen considers a class of nonsmooth generalized semi-infinite programming problems, where estimates for the value functions of the lower level problems and necessary optimality conditions for generalized semi-infinite programming problems are derived.

Finally, hybrid and relaxed Mann iteration methods for a general system of variational inequalities with solutions being also common solutions of a countable family of variational inequalities and common fixed points of a countable family of nonexpansive mappings in real smooth and uniformly convex Banach spaces are proposed in the paper by L. C. Ceng et al., where they derive some strong convergence theorems for hybrid and relaxed Mann iteration algorithms not only in the setting of uniformly convex and 2-uniformly smooth Banach space but also in a uniformly convex Banach space having a uniformly Gateaux differentiable norm.

\section{Acknowledgments}

The editors of this special issue would like to express their gratitude to the authors who have submitted their manuscripts for consideration and to the many expert referees who were involved in the reviewing process and provided us an invaluable help. 


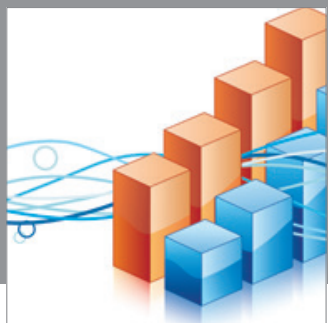

Advances in

Operations Research

mansans

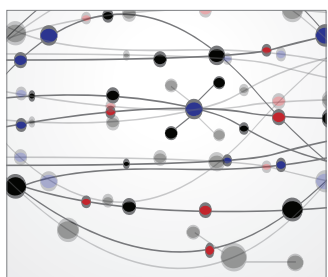

The Scientific World Journal
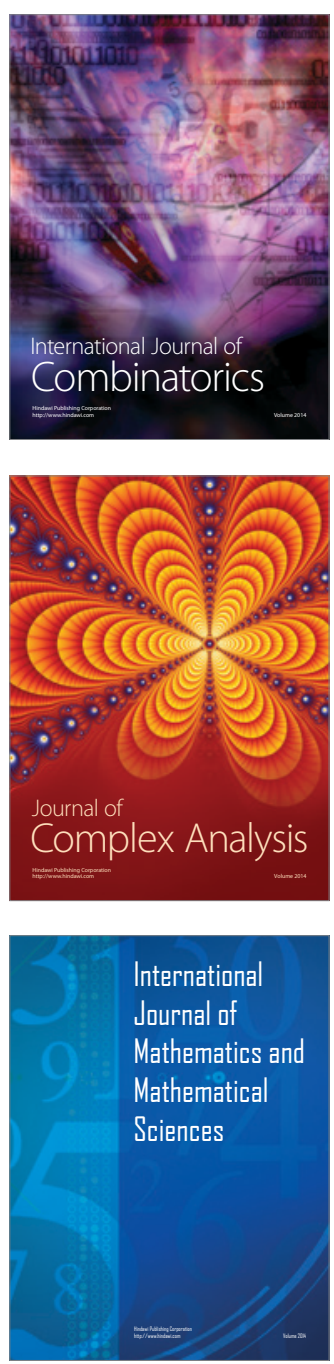
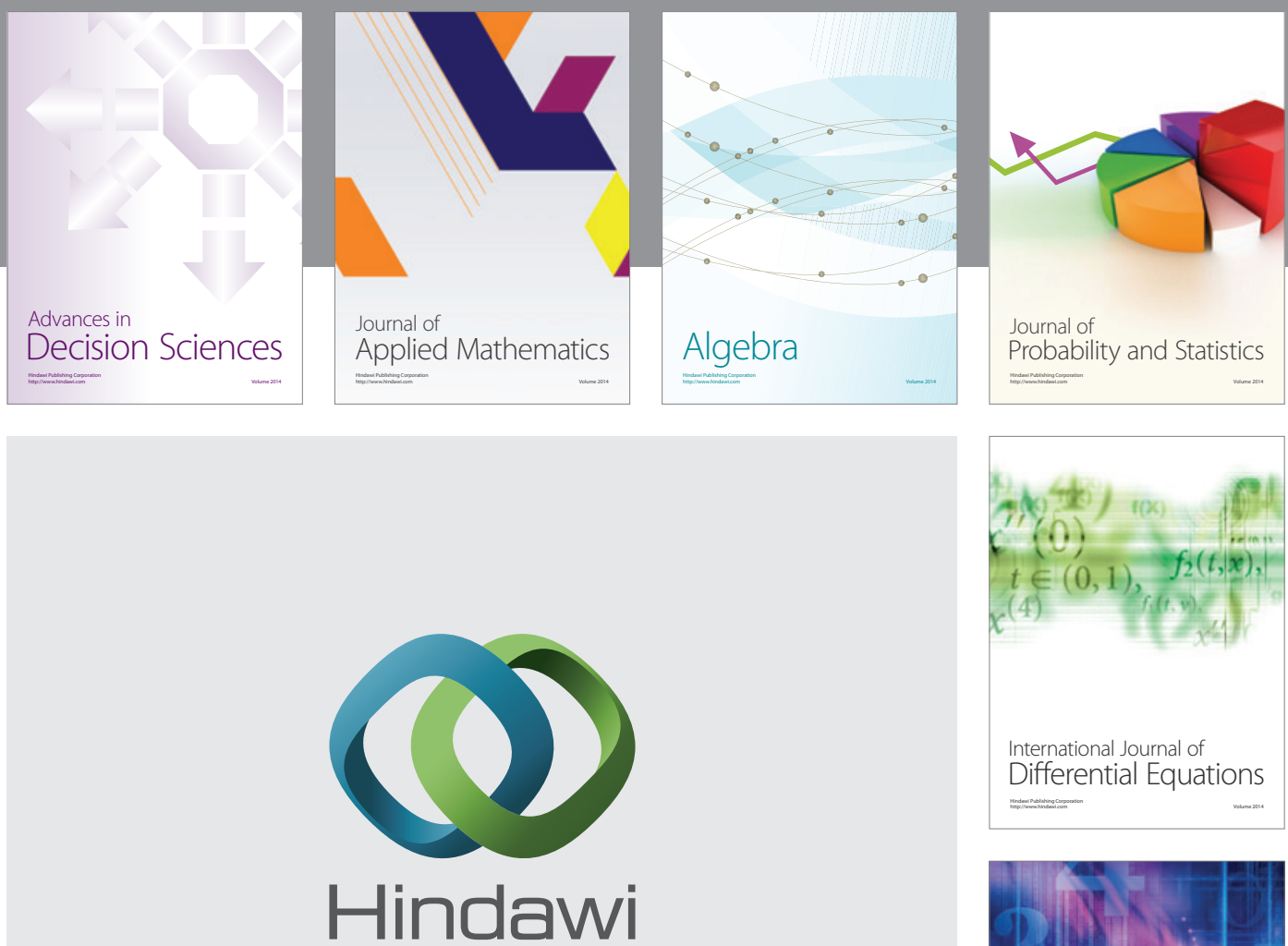

Submit your manuscripts at http://www.hindawi.com
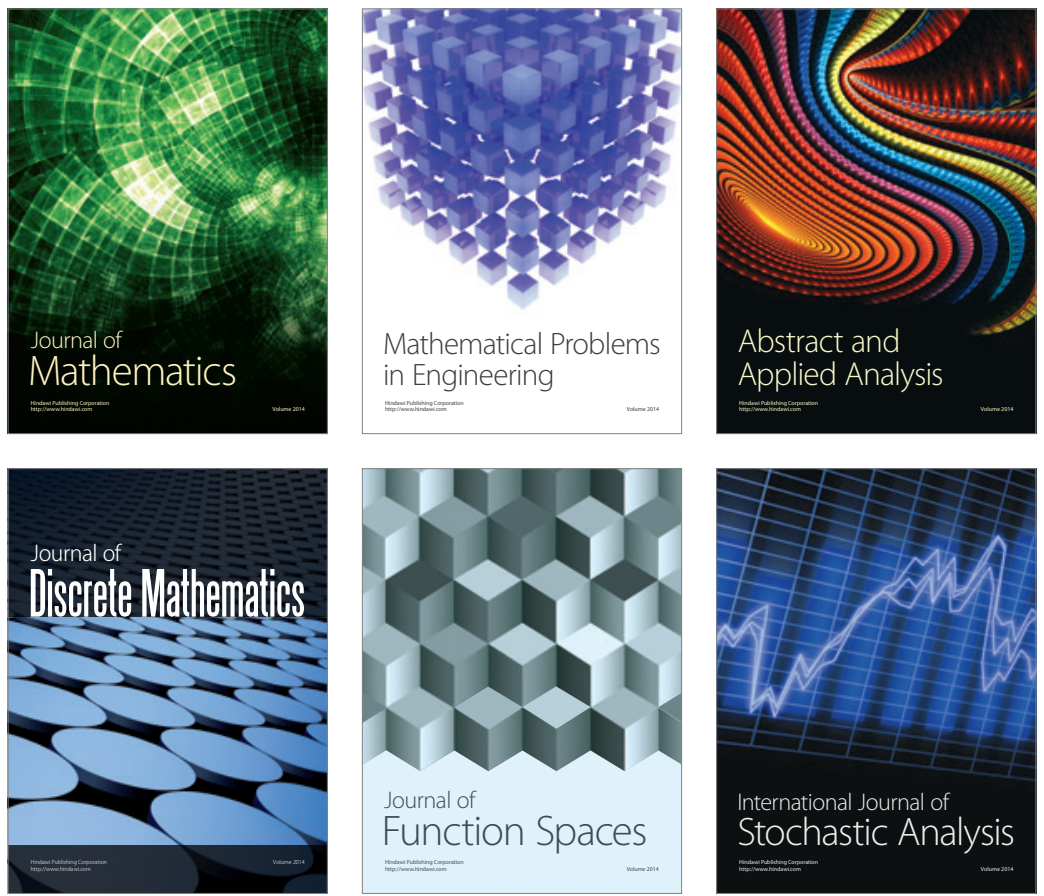

Journal of

Function Spaces

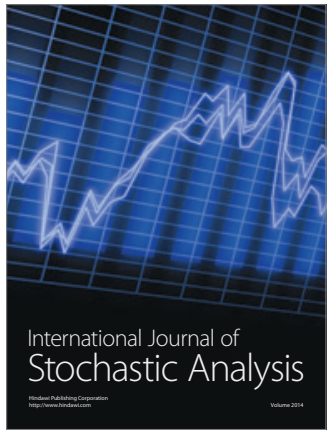

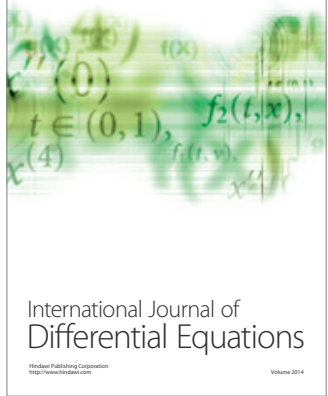
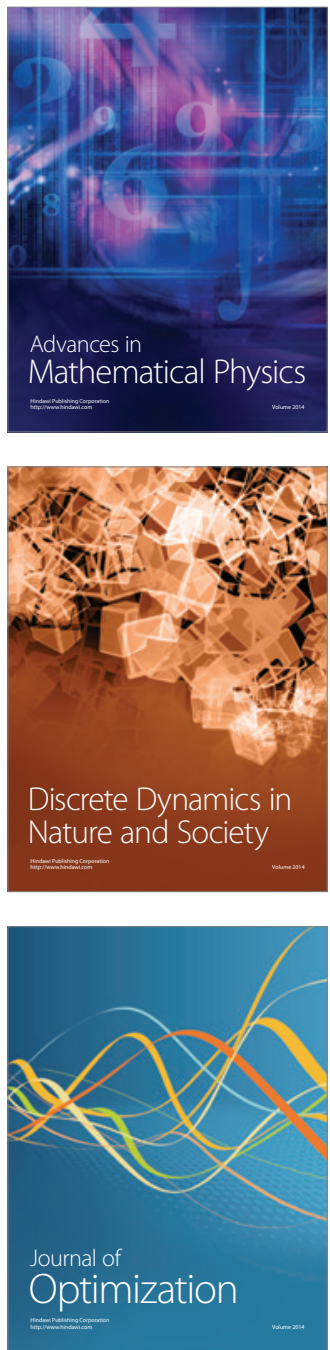